\title{
Development of Parkinson and Alzheimer Diseases in Two Cases of Narcolepsy-Cataplexy
}

\author{
Nicholas-Tiberio Economou ${ }^{a}$ Mauro Manconi ${ }^{a} \quad$ Joseph Ghika ${ }^{b}$ Monika Raimondi ${ }^{a}$ \\ Claudio L. Bassetti ${ }^{a}$ \\ ${ }^{a}$ Neurocenter of Southern Switzerland, Lugano, and ${ }^{b}$ Neurology Division, CHCVs, Hôpital de Sion, \\ Sion, Switzerland
}

Dear Sir,

Narcolepsy (NC) is a sporadic hypersomnia (prevalence 1:2,000), characterized by excessive daytime sleepiness (EDS) and sleep attacks, typically associated with cataplexy and other REM-sleep related phenomena such as sleep paralysis and hallucinations [1]. In human NC with cataplexy, an association with specific HLA haplotype (DR DQB1 0602) and a deficiency in hypocretin (orexin) peptide in cerebrospinal fluid (CSF) are almost constant findings. The pathogenesis of human NC is still unknown. Autoimmune and neurodegenerative processes of hypothalamic structures have been discussed with more solid evidence for the first theory [2-4]. Most of narcoleptic symptoms develop early after the onset of the disease and usually do not worsen with the progression of the neurodegeneration [2]. Moreover, the absence of ubiquitinated inclusions (cardinal neuropathological finding of most neurodegenerative diseases) in narcoleptic patients argues against the neurodegenerative hypothesis in NC [3]. An autoimmune process involving the hypocretin neurons of lateral hypothalamus has been postulated [4].

There are few reports in the literature showing the occurrence of NC-like symptoms in patients already affected by Parkinson's disease (PD) and other neurodegenerative diseases [2, 5-7]. Hypocretin neuronal loss has been documented in subjects with advanced PD and Alzheimer's disease $(\mathrm{AD})[6,7]$. A possible relationship between neurodegenerative disorders, especially PD as a possible cause of typical NC with hypocretin deficiency and HLA haplotype, and NC-like phenotype is still under discussion.

Herein, we describe 2 patients with a long history of $\mathrm{NC}$ who developed PD and $\mathrm{AD}$, respectively.

\section{Case Reports}

Case 1

A 69-year-old Caucasian male, suffering from NC since the age of 17, presented to us at the age of 64 years (in 2006). His history included EDS with sleep attacks, hallucinations, frequent cataplexy and occasional sleep paralysis. Patient's psychomotor development was normal and clinical history unremarkable; in particular, he denied infectious or inflammatory cerebral diseases, head trauma and exposure to toxic agents.

During his NC history, the patient underwent several drug treatments (methylphenidate, modafinil, dexedrine, imipramine) with partial benefit on EDS and cataplexy.

At that time, he presented the typical HLA haplotype (HLA DQB1*0602), unde- tectable CSF hypocretin 1 values and typical neurophysiological findings (MSLT with a sleep latency, SL, of 6.6' and 4 sleep onset REM periods - SOREMPs). At the time of our evaluation, he was already on modafinil and imipramine and presented a resting tremor, micrographia, and slight bradykinesia of the right hand. Hyposmia was documented with olfactory tests. Total Unified Parkinson's Disease Rating Scale (UPDRS III) score was 13 (mental activities: 0 , activities of daily living: 6 , motor: 7; UPDRS III range is 0-176). The brain MRI was normal. Before our evaluation, the tremor was diagnosed as essential (he had a family history of essential tremor) and specific treatments (beta-blockers, primidone and anticholinergic agents - biperiden) were already tried without efficacy. Conversely, cataplexy was partially improved by the anticholinergic treatment (biperiden), while other narcoleptic symptoms remained unchanged. Because of the limited response to the therapy, the patient was started on L-dopa-benserazide (until the final dose of 1,000 mg/day), which ameliorated all symptoms, with a decrease of $70 \%$ in motor UPDRS score (total score: 3 , mental activities: 0 , activities of daily living: 1, motor: 2). Thus, according to the standard diagnostic criteria, the diagnosis of PD was done.

\section{KARGER}

Fax +41613061234 E-Mail karger@karger.ch www.karger.com
Dr. Mauro Manconi

Neurology Department, Neurocenter of Southern Switzerland

Via Tesserete 46

CH-6900 Lugano (Switzerland)

Tel.+41 091811 6825,E-Mail mauro.manconi@eoc.ch 
Table 1. Clinical features before and after neurodegenerative disorder development

\begin{tabular}{|c|c|c|c|c|c|}
\hline \multirow[t]{2}{*}{ Clinical feature } & \multicolumn{2}{|l|}{ PD patient } & \multicolumn{3}{|l|}{$\mathrm{AD}$ patient } \\
\hline & $\begin{array}{l}\text { before PD } \\
\text { development }\end{array}$ & $\begin{array}{l}4 \text { years after PD } \\
\text { development }\end{array}$ & $\begin{array}{l}\text { before AD } \\
\text { development }\end{array}$ & $\begin{array}{l}4 \text { years after AD } \\
\text { development }\end{array}$ & $\begin{array}{l}6 \text { years after } \mathrm{AD} \\
\text { development }\end{array}$ \\
\hline EDS & severe (ESS 20) & moderate (ESS 11) & severe (ESS 16-18) & slight (ESS 8) & n.a. \\
\hline Mean SL, min & 6.6 (treated) & n.a. & 1.9 & 4.5 & 1 \\
\hline Cataplexy & severe, daily & rare, only eyelid & daily & no & no \\
\hline SOREM & 4 & n.a. & 2 & 2 & 5 \\
\hline Sleep paralysis & yes & rare & no & no & no \\
\hline Hallucinations & yes & rare & frequent & rare & frequent \\
\hline CSF Hyp-1 & undetectable & - & undetectable & - & - \\
\hline HLA DQB1*0602 & positive & - & positive & - & - \\
\hline
\end{tabular}

ESS = Epworth Sleepiness Scale; Hyp-1 = hypocretin; n.a. = not available.

Soon after the introduction of L-DOPA, symptoms of NC also drastically improved, in particular cataplexy, which remitted completely with the exception of episodes of short duration of eyelid ptosis on emotional triggers; EDS improved as well (Epworth Sleepiness Scale 11/24). The above-described NC status remained unchanged until the last clinical assessment (at the age of 68, in 2010).

Case 2

A 71-year-old Caucasian male suffered from NC symptoms since his adolescence. He always claimed severe EDS with recurrent sleep attacks. Frequent cataplectic episodes were also experienced since adolescence, usually triggered by positive emotions (mainly laughing). Insomnia (fragmented sleep) and hypnopompic hallucinations but no sleep paralysis were reported. Because of the severe disability, the patient was exempted from his military duty and preferred working at home as a painter, adjusting his daily activities to his symptoms. Based on clinical and neurophysiological findings (2 SOREMPs, mean SL of $2^{\prime}$ at the MSLT), the diagnosis of NC was made at the age of 62 (in 2002), and further confirmed at 69 (in 2009; MSLT: 2 SOREMPs, mean SL $4.5^{\prime}$ ). The HLA typing was positive (HLA DQB1*0602), while the CSF hypocretin 1 was undetectable. His medical history was positive for depression, parasomnia and snoring. Two video-PSGs followed by MSLTs, performed at the age of 62 and 69, respectively, revealed night gesturing, and talking during REM sleep in a context of a dream- like behavior. A mixed (central and obstructive) sleep breathing disorder was also observed (AHI was 23 at 62 and 43 at 69 years). After NC was diagnosed, modafinil (200 mg/day) and venlaflaxine (75 mg/day) for both cataplexy and depression were introduced. Both drugs were effective in improving symptoms. At the age of 65 (in 2005), the patient developed progressive cognitive decline, characterized by spatial disorientation, short-term memory deficit, reduced attention and verbal fluency; the MMSE was 23/30. Brain MRI showed generalized cortical atrophy, involving temporoparietal and hippocampal cortex without focal lesions. According to standard criteria, a clinical diagnosis of probable AD was made. His mother had also suffered from late-onset dementia. The patient received rivastigmine with scarce improvement. As AD progressed (current MMSE score 5/30), paraphasias and neologisms appeared and the therapy was switched to galantamine (24 mg/day). His NC symptoms subjectively improved (mild subjective EDS, cataplexy improved as well). The patient was last seen at the age of 69 (in 2011), when his last MSLT demonstrated mean SL of $1 \mathrm{~min}$ and 5 SOREMPs. CSF measurements of Tau-protein and $\beta$ amyloid are currently pending.

\section{Discussion}

While the development of NC-like syndromes secondary to neurodegenerative disorders (mostly PD) is well described in the literature, there are only isolated re- ports regarding the appearance of neurodegenerative disorders in subjects already affected by NC $[3,7]$. Especially as far as $\mathrm{AD}$ is concerned, the reports in the literature document postmortem diagnosis of $\mathrm{AD}$. Thus, our patient is the first one reported suffering from NC in whom diagnosis of $\mathrm{AD}$ according to the international criteria was done when still alive, receiving consequently the appropriate therapy.

The prevalence of PD and AD at the age and in the geographic area of the 2 patients described here, is estimated at 9.5/1,000 for PD and 48/1,000 for AD, respectively [8], while the prevalence of $\mathrm{NC}$ is around $0.5 / 1,000$ [1]. Thus, the probability for NC patients to present comorbid $\mathrm{PD}$ and $\mathrm{AD}$ can be estimated at about 4.75/1,000,000 and $24 / 1,000,000$, respectively. Although infrequent, these proportions are not extremely rare (especially when considering referral centers for NC), and may suggest a rare but not negligible comorbidity. A recent neuropathological study on $12 \mathrm{NC}$ patients showed a similar prevalence of AD among them compared to the prevalence of $\mathrm{AD}$ in the general population [7]. Although no available evidence of a predisposition of NC to neurodegenerative diseases (PD and $\mathrm{AD}$ in particular) exists, given that both dopaminergic and cholinergic systems are implicated in the sleep/ wake regulation, some considerations regarding their role, their dysregulation and also their complex interactions with the hypocretin neuronal networks might be discussed.

Hypocretin deficiency due to hypocretin cell loss in lateral hypothalamus is the 
hallmark neuropathological damage in NC. A partial hypocretin deficiency seems to be also common in PD and was recently shown in an $\mathrm{AD}$ postmortem study as well $[6,7]$. In PD, an association with narcoleptic-like symptoms, such as dreaming-like phenomena while awake, hypnagogic hallucinations and REM behavior disorder, which often precede the appearance of motor symptoms of $\mathrm{PD}$, is not a rare phenomenon, suggesting a progressive hypocretin cell loss of degenerative origin.

In the first patient described here, the PD onset was not followed by a worsening of the narcoleptic symptoms. Interestingly, the dopaminergic treatment improved EDS and cataplexy allowing the withdrawal of the NC medication. This finding is in contrast with the common knowledge regarding dopaminergic treatments, which not only do not enhance vigilance, but also may induce sleep attacks both in PD patients and in healthy controls [9-11].

Sleep-wake pattern abnormalities in PD patients could depend on both hypocretin deficiency and dopaminergic dysfunction. In fact, the dopaminergic neurons play an important role in promoting wakefulness. An impairment of the dopaminergic system has also been suggested in $\mathrm{NC}$, for instance $\mathrm{D}_{2}$-receptor binding was elevated in NC and positively correlated with the frequency of cataplectic and sleep attacks. Furthermore, an impairment of the dopamine-mediated reward system in NC has also been described [1214]. This observation suggests a complex interaction between dopaminergic and hypocretin neuronal networks, which needs further investigations.

Acetylcholine plays an important role in promoting vigilance toward a diffuse cortical activation. A cholinergic deficit is a hallmark of AD. Dysfunction in orexinacetylcholine interactions may play a role in the arousal and attention deficits seen in neurodegenerative conditions, in drug addiction and in age-related cognitive decline as well [15]. Moreover, data from canine NC studies suggested that a primary deficit in orexin signaling might contribute to postsynaptic degeneration and affect Ach-dependent cognitive functions [16]. Under this prospective, it would be expected that the occurrence of a neurodegenerative disorder could worsen at least some of the NC symptoms, especially EDS. Conversely, in both of our patients the appearance of neurodegenerative process was followed by a significant improvement of all narcoleptic symptoms, including EDS and cataplexy. EDS's paradoxical improvement, as already reported [17], may be due to the overall sleep improvement and sleep-wake cycle regulation following cholinergic treatment. Interestingly, cataplexy is also mediated by the cholinergic system, and usually responds to anti-cholinergic drugs, while in our case, cataplexy was improved by an enhancement of the cholinergic system. This observation together with the overall amelioration of the narcoleptic symptoms after the clinical onset of the neurodegenerative diseases confirms the complexity of the interactions between the hypocretin/dopaminergic and cholinergic systems.

In this scenario, a long-term follow-up of narcoleptic patients becomes extremely relevant to better understand the abovementioned complex neuronal network interactions and to improve the therapeutic approach in narcoleptic patients with neurodegenerative comorbidity.

\section{References}

1 American Academy of Sleep Medicine: International Classification of Sleep Disorders: Diagnostic and Coding Manual, ed 2. Westchester, American Academy of Sleep Medicine, 2005.

-2 Baumann C, Ferini-Strambi L, Waldvogel D, Werth E, Bassetti CL: Parkinsonism with excessive daytime sleepiness - a narcolepsylike disorder? J Neurol 2005;252:139-145.

3 Honda M, Arai T, Fukazawa M, Honda Y, et al: Absence of ubiquitinated inclusions in hypocretin neurons of patients with narcolepsy. Neurology 2009;73:511-517.

-4 Fontana A, Gast H, Reith W, Recher M, et al: Narcolepsy: autoimmunity, effector T cell activation due to infection, or T cell independent, major histocompatibility complex class II induced neuronal loss? Brain 2010;133: 1300-1311.

5 Fronczek R, Overeem S, Lee SY, Hegeman IM, et al: Hypocretin (orexin) loss in Parkinson's disease. Brain 2007;130:1577-1585.

-6 Fronczek R, Baumann CR, Lammers GJ, Bassetti CL, et al: Hypocretin/orexin disturbances in neurological disorders. Sleep Med Rev 2009;13:9-22.

7 Scammell TE, Matheson JK, Honda M, Thannickal TC, Siegel JM: Coexistence of narcolepsy and Alzheimer's disease. Neurobiol Aging, Epub ahead of print.

8 Hirtz D, Thurman DJ, Gwinn-Hardy K, Mohamed M, et al: How common are the 'common' neurologic disorders? Neurology 2009; 68:326-337.

$\checkmark 9$ Hobson DE, Lang AE, Martin WR, Razmy A, et al: Excessive daytime sleepiness and sudden-onset sleep in Parkinson disease: a survey by the Canadian Movement Disorders Group. JAMA 2002;287:455-463.

10 Micallef J, Rey M, Eusebio A, Audebert C, et al: Antiparkinsonian drug-induced sleepiness: a double-blind placebo-controlled study of L-dopa, bromocriptine and pramipexole in healthy subjects. Br J Clin Pharmacol 2009;67:333-340.

11 Boivin DB, Montplaisir J: The effects of Ldopa on excessive daytime sleepiness in narcolepsy. Neurology 1991;41:1267-1269.
12 Poryazowa R, Benninger D, Waldvogel D, Bassetti CL: Excessive daytime sleepiness in Parkinson's disease: characteristics and determinants. Eur Neurol 2010;63:129-135.

13 Nishino S, Arrigoni J, Valtier D, Miller JD, et al: Dopamine mechanisms in canine narcolepsy. J Neurosci 1991;11:2666-2671.

-14 Ponz A, Khatami R, Poryazowa R, Werth E, et al: Abnormal activity in reward brain circuits in human narcolepsy with cataplexy. Ann Neurol 2010;67:190-200.

15 Fadela J, Burk JA: Orexin/hypocretin modulation of the basal forebrain cholinergic system: role in attention. Brain Res 2010;1314: 112-123.

16 Siegel JM, Nienhuis R, Gulyani S, Ouyang S, et al: Neuronal degeneration in canine narcolepsy. J Neurosci 1999;19:248-257.

17 Nieoullon A, Bentue-Ferrer D, Bordet R, Tsolaki $\mathrm{M}$, et al: Importance of circadian rhytmicity in the cholinergic treatment of Alzheimer's disease: focus on galantamine. Curr Med Res Opin 2008;24:3357-3367. 